\title{
Physical Exercise Engagement: Its Impact on Mental Health to the Tertiary Students Amidst COVID-19 Pandemic
}

\author{
Vanessa S. Maghanoy \\ vanessa.salomon-maghanoy@g.msuiit.edu.ph \\ Mindanao State University-Iligan Institute of Technology, Iligan City, 9200, Philippines
}

\begin{abstract}
The COVID-19 pandemic has become a worldwide public health concern. People experienced a prevalent decline in well-being, retrogression in mental health, and psychological distress such as stress, anxiety, depression, and feelings of isolation. Physical activity has traditionally been paramount in treating mental health issues. This study aimed to assess the impact of physical exercise engagement among the tertiary students' mental health in MSU-IIT amidst the COVID-19 pandemic. A total of 300 respondents participated in the descriptive-quantitative research study. Based on the study result, the respondents engaged in moderate to light physical activity for 30-60 minutes each day during and outside their P.E. classes. Dance, jogging, stretching, and sports are the physical exercise engagement of the respondents. Significant correlation of physical exercise engagement to the mental health status of the respondents reveals that physical exercise has a low negative correlation to psychological distress at $\mathrm{r}=-0.481$, and there is a significant relationship at $\mathrm{p}=0.000$. It also shows that physical exercise has a moderate negative correlation to depression severity at $\mathrm{r}=-0.619$, and there is a significant relationship at $\mathrm{p}=0.000$. It further reveals that physical exercise has a moderate negative correlation to anxiety disorder at $\mathrm{r}=-0.699$, and there is a significant relationship at $\mathrm{p}=0.000$. It further implies that increasing physical activity will help stimulate and reduce the psychological suffering response of mental health. Thus, it is recommended that physical exercise succour and benefit everyone's mental health.
\end{abstract}

Keywords: Physical Exercise Engagement; Mental Health; Students

\section{Introduction}

The COVID-19 pandemic has been felt worldwide since the beginning of 2020 and has disrupted many aspects of life, including schools. Uncertainty surrounds the timing of school closures in March 2020 and the impact of the current academic year's almost complete shift to online learning. This outbreak has brought attention to the affected communities' mental health. It has been linked to fear for oneself or loved ones and restrictions on physical and social activities due to quarantine. Current outbreaks and pandemics were detected by stressors (i.e., infection fear, dissatisfaction, boredom, lack of resources, information, financial loss, and stigma). Advocates for Education said the prolonged closure of schools had a significant impact on student's development and mental health. For many students, disadvantaged and poorest communities, in particular, some parents are uneducated, others are too focused on work due to financial stress, and the school can be 
a place where students can have a chance to learn (theguardian.com, 2021). If the situation continues, many students will eventually lose interest in school or be forced to drop out.

WHO-led study says most adolescents worldwide are not sufficiently physically active, putting their current and future health at risk (World Health Organization, 2019). Regular exercise has been demonstrated to improve one's health. Aerobic capacity and muscle size are not the only concerns. Surely, exercise can improve one's health, body, sex life and even add years to one's life, but that does not keep most people engaged. Regular exercise promotes a sense of well-being. More energy, better sleep, stronger memory, and a calmer, happier outlook on life are all effects of feeling invigorated throughout the day (Weil, 2021).

According to a study, exercise is also a very effective treatment for many mental health conditions. Studies show that exercise effectively reduces fatigue, improves alertness and concentration, and enhances overall cognitive function. This can be helpful significantly when stress has depleted one's energy or ability to concentrate. Physical activity and other exercises produce endorphins - chemicals in the brain that act as natural painkillers - and improve the ability to sleep, reducing stress (ADAA, 2022). Exercise helps with depression, anxiety, and ADHD (Lawalata, 2021). It can alleviate stress, develop memory, enhance sleep, and improve mood. Getting fit does not require being a fitness nut. A little exercise can make a big difference. Regardless of one's age or fitness level, one can get more out of life by exercising to improve one's mental health, vitality, and outlook (Robinson et al., 2020).

Amidst the Covid-19 pandemic, home quarantines and physical activity restrictions have disrupted the communities' mental health and physical activity status. Hence, physical activity is crucial and must be well integrated into everyone's daily work as it enhances one's physical health and positively impacts one's mental health. Students of all levels, workers, teachers, and even regular people must engage in various physical activities. To the researchers' knowledge, there were only a few studies had explored the effects of a pandemic on the students' mental health in MSU-IIT, as they also require special care and concern, considering their vulnerability during the pandemic. Specifically, the researcher aims to investigate the impact of physical exercise on the students' mental health in MSU-IIT during the COVID-19 pandemic.

2.

\section{Methodology}

This study used descriptive-quantitative method of research. A snowball sampling procedure was implemented in the study in which an existing respondent provided referrals to recruit other respondents whose qualities deemed fit based on the requirement described in the respondents/participants of the study section supported with standardized questionnaire distributed through google form for data gathering of the study. The instrument has three (3) parts. First, the socio-demographic profile of the respondents, second is the Physical Activity Engagement of the students and third, are three adapted standard questionnaires, the Kessler Distress Scale, Patient Health Questionnaire, and the Generalized Anxiety Disorder Scale. 300 enrolled students of MSUIIT in A.Y. 2021-2022 participated in this research. This study describes participants' response to the questions provided to them by the researchers in order to gather significant information. According to Ritchey and Klein (2005), this method was appropriate because descriptive statistics utilized data collection and analysis techniques to yield reports concerning central tendency, variation, and correlation. Quantitative method pertains to the number of students that pick their answer in each question and will be counted as part of data gathering and information. 
Descriptive statistics were used in the first part of the analysis for the computation of mean, standard deviation, and percentages to describe the participants' demographic characteristics. Correlation analysis was performed to determine the relationship among the variables.

To guarantee that ethical considerations were taken into account, the participants' confidentiality and anonymity were maintained as they were given choice of filling in their names. The researchers provided informed consent to the respondents ahead of time to ensure their approval of participation during the data collection.

\section{Results and Discussion}

3.1 Physical Exercise Engagement of the Respondents Amidst COVID-19 Pandemic

Table 3.1.1 Physical Exercise Engagement of the Respondents during P.E class

\begin{tabular}{|c|c|c|}
\hline Physical Exercise Engagement & Frequency & Percentage \\
\hline \multicolumn{3}{|c|}{ Engagement } \\
\hline Yes & 300 & $100 \%$ \\
\hline No & 0 & $0 \%$ \\
\hline Total & 300 & $100 \%$ \\
\hline \multicolumn{3}{|c|}{ Frequency } \\
\hline Once & 300 & $100 \%$ \\
\hline 2-3 times & 0 & $0 \%$ \\
\hline 4-5 times or more & 0 & $0 \%$ \\
\hline Total & 300 & $100 \%$ \\
\hline \multicolumn{3}{|c|}{ Intensity } \\
\hline Light & 114 & $38 \%$ \\
\hline Moderate & 180 & $60 \%$ \\
\hline Heavy & 6 & $2 \%$ \\
\hline Total & 300 & $100 \%$ \\
\hline \multicolumn{3}{|c|}{ Time Spent } \\
\hline 15-29 minutes & 27 & $9 \%$ \\
\hline 30-45 minutes & 105 & $35 \%$ \\
\hline 45-60 minutes & 156 & $52 \%$ \\
\hline 1 hour or more & 12 & $4 \%$ \\
\hline Total & 300 & $100 \%$ \\
\hline \multicolumn{3}{|c|}{ Types of Activities } \\
\hline Swimming & 2 & $1 \%$ \\
\hline Jogging & 60 & $20 \%$ \\
\hline Sports & 47 & $16 \%$ \\
\hline Dancing & 67 & $22 \%$ \\
\hline Stretching & 88 & $29 \%$ \\
\hline Walking & 36 & $12 \%$ \\
\hline Total & 300 & $100 \%$ \\
\hline
\end{tabular}


Table 3.1.2 Physical Exercise Engagement of the Respondents outside P.E class

\begin{tabular}{|c|c|c|}
\hline \multicolumn{3}{|l|}{ OUTSIDE P.E. CLASS } \\
\hline \multicolumn{3}{|c|}{ Engagement } \\
\hline Yes & 291 & $97 \%$ \\
\hline No & 9 & $3 \%$ \\
\hline Total & 300 & $100 \%$ \\
\hline \multicolumn{3}{|c|}{ Frequency } \\
\hline Once & 81 & $27 \%$ \\
\hline 2-3 times & 204 & $68 \%$ \\
\hline 4-5 times or more & 15 & $5 \%$ \\
\hline Total & 300 & $100 \%$ \\
\hline \multicolumn{3}{|c|}{ Intensity } \\
\hline Light & 108 & $36 \%$ \\
\hline Moderate & 180 & $60 \%$ \\
\hline Heavy & 12 & $4 \%$ \\
\hline Total & 300 & $100 \%$ \\
\hline \multicolumn{3}{|c|}{ Time Spent } \\
\hline 15-29 minutes & 39 & $13 \%$ \\
\hline 30-45 minutes & 147 & $49 \%$ \\
\hline 45-60 minutes & 93 & $31 \%$ \\
\hline 1 hour or more & 21 & $7 \%$ \\
\hline Total & 300 & $100 \%$ \\
\hline \multicolumn{3}{|c|}{ Types of Activities } \\
\hline Swimming & 6 & $2 \%$ \\
\hline Jogging & 75 & $25 \%$ \\
\hline Sports & 45 & $15 \%$ \\
\hline Dancing & 81 & $27 \%$ \\
\hline Stretching & 57 & $19 \%$ \\
\hline Walking & 36 & $12 \%$ \\
\hline
\end{tabular}

Table 3.1.1 shows the frequency and percentage distribution of physical exercise engagement of the participants during P.E. class. In terms of the respondents' physical exercise engagement, all of the respondents engaged in physical exercise once a week during their P.E. class. It can be observed that in the total number of respondents, 180 or $60 \%$ selected a moderate intensity level of exercise, while 114 or $38 \%$ is a light intensity level. Among the 300 respondents, 156 or $52 \%$ engaged in physical exercise for 45-60 minutes, and 105 or 35\% was 30-45 minutes time spent. It can be noticed that 88 or $29 \%$ of respondents selected stretching as the type of physical activity they engaged in, 67 (22\%) in dance while on jogging is 60 or $20 \%$, and 47 or $16 \%$ chose the sports activity.

Table 3.1.2 shows the frequency and percentage distribution of physical exercise engagement of the participants outside P.E. class. In terms of the respondents' physical exercise engagement, 291 or $97 \%$ of the respondents engaged in physical exercise, usually 2-3 times in a week aside from their P.E. class, 81 or $27 \%$ engaged once in a week while 15 or $5 \%$ engaged in 
physical activity for $4-5$ times. It can be observed that in the total number of respondents, 180 or $60 \%$ selected a moderate intensity level of exercise, while 108 or $36 \%$ is light intensity level while there are $4 \%$ or 12 respondents performed heavy intensity of physical activity. Among the 300 respondents, 147 or $49 \%$ engaged in physical exercise for $30-45$ minutes and 93 or $31 \%$ is $45-60$ minutes time spent, $13 \%$ or 39 respondents engaged for $15-29$ minutes and $7 \%$ or 21 of the respondents engaged for 1 hour or more. It can be noticed that 81 or $27 \%$ of respondents selected dancing as the type of physical activity they engaged in, 75 (25\%) in jogging while on stretching is 57 or $19 \%$ and 45 or $15 \%$ choose the sports activity and there are at least $12 \%$ or 36 of the respondents walked.

Students earned physical education basic courses that promote an understanding of the centrality of movement in daily life, in all its forms, acknowledging physical activity and sports participation as significant cultural and health practices in the Philippines, developing school loyalty and nationalism through the sporting culture, which in turn significantly shapes an individual's identity. It is included in the curriculum with equivalent to 2 units/ 2 hours of class per session/ week in one semester. Aside from the students being active during the P.E. class, most of them also engage in physical activities outside P.E. class.

According to the Affective-Reflective Theory (ART) of physical inactivity and exercise (Brand and Ekkekakis, 2018), stimuli trigger automatic associations resulting in an automatic affective valuation of exercise. The ART aimed to explain and predict behaviour in situations in which an individual either remains in a state of physical inactivity or initiates the action and assumes that experience, feelings, and thoughts connected with exercise affect whether an individual is eager to undergo the same physical strain experienced on the previous exercise. Teachers and parents should promote an active and healthy lifestyle for children and adolescents to support this claim. Although every school subject should promote healthy activities, Physical Education (P.E.) should spearhead in fostering well-being habits associated with a healthy lifestyle during sports practice and other physical activities (Frontiers in Psychology, 2019).

The ART theory explains as an automatic affective valuation, an unattended assignment of positive (association with pleasure) or negative (association with displeasure) value to a stimulus, either as the result of repeated exercise-related emotional experiences mediated by cognitive appraisals (e.g., pride, embarrassment) or as a result of repeated experiences of core affective reactions to stimuli (e.g., sense of physical reinvigoration, bodily discomfort). Refer Table 3.1.1 and 3.1.2 Thus, the participants perform exercise intensity is moderate $(\mathrm{N}=180,60 \%)$ and did it frequently, mostly $2-3$ times a day $(\mathrm{N}=204,68 \%)$ in the activities they mostly perform. Most respondents spent up to $30-60$ minutes $(\mathrm{N}=240,80 \%)$ of their time doing their routine inside and outside P.E. Class, and we can see that most of the respondents were really motivated and energetically engaged at this age range. Tertiary students have much energy during physical activity to perform much (Physical Education Class XI, CBSE, Delhi, 2020). The most performed activities were dance, jogging, stretching, sports, also walking. Few of them do swimming activities as most of them can be easily performed without equipment. Their P.E. teachers have already led the instructions and activities to perform. It may help warm up the body or get the muscles moving and ready to work (Warburton, 2006). The results show positive emotional experiences triggered by the satisfaction of autonomy, pleasantness, and more activating. According to the Article, Students' Emotional Experience in Physical Education-A Qualitative Study for New Theoretical Insights (Sascha Leisterer et al., 2019), a teacher asks the students to express their wishes and ideas for the P.E. classes. With this democratic approach, novelty leads to perceived autonomy. Autonomy 
triggers positive experiences, and as one student called Marie states: "this is a great thing to happen in every P.E. lesson. I am always looking forward to our P.E. classes". We cannot contrast autonomy as a trigger of positive emotions with a negative experience with our data. No interviewee reported adverse effects concerning autonomy. Autonomy supportive situations in P.E. attract students to a specific task. Mainly, variety and novelty influence these situations positively.

Overall, as observed, the study shows the respondent's level of physical exercise engagement scores is 183 or $61 \%$, which fall under the average level of physical exercise engagement. Engagement in physical education classes benefits cardiorespiratory fitness and muscle strength seen among students who practiced sports during the class (Henrique et al., 2017). Moderate to vigorous physical activity and exercise during the day is associated with elevated self-esteem. Also, it improves concentration, reductions in depressive symptoms, and improvements in sleep. Additionally, participation in regular physical activity boosts the immune system.

3.2 Mental Health Status of the Respondents as Measured According to Kessler Psychological Distress Scale, Patient Health and Generalized Anxiety Disorder Scale

Table 3.2.1 Mental Health Status of the Respondents when measured according to Kessler Psychological Distress Scale, Patient Health and Generalized Anxiety Disorder Scale

\begin{tabular}{|c|c|c|}
\hline Mental Health Status & $\mathrm{N}=300$ & Percentage \\
\hline \multicolumn{3}{|c|}{ Psychological Distress } \\
\hline Likely to be well & 249 & $83 \%$ \\
\hline Likely to have a mild disorder & 51 & $17 \%$ \\
\hline Likely to have a moderate disorder & 0 & $0 \%$ \\
\hline Likely to have a severe disorder & 0 & $0 \%$ \\
\hline Total & 300 & $100 \%$ \\
\hline \multicolumn{3}{|c|}{ Depression } \\
\hline no symptoms & 54 & $18 \%$ \\
\hline Mild & 222 & $74 \%$ \\
\hline Moderate & 24 & $8 \%$ \\
\hline moderately severe & 0 & $0 \%$ \\
\hline Severe & 0 & $0 \%$ \\
\hline Total & 300 & $100 \%$ \\
\hline \multicolumn{3}{|c|}{ Anxiety } \\
\hline minimal anxiety & 63 & $21 \%$ \\
\hline mild anxiety & 174 & $58 \%$ \\
\hline moderate anxiety & 60 & $20 \%$ \\
\hline Total & 300 & $100 \%$ \\
\hline
\end{tabular}

The table 3.2.1 shows the mental health status of the respondents. In terms of the psychological distress, $249(83 \%)$ of the respondents are likely to be well and 51 or $17 \%$ are likely to have a mild disorder. It can be observed that 222 or $74 \%$ of the respondents have mild depression and no one fall in the severe. In terms of the anxiety level, the result shows that $174(58 \%)$ of the respondents have mild anxiety and $60(20 \%)$ have moderate anxiety. 
Participants were directed to choose the sentence that best described their emotions in the Kessler Psychological Distress Scale over the past month. It was found that the status of the respondents is likely to be well $(\mathrm{N}=249,83 \%)$. The average participants' score of 18 explains that they are not affected when it comes to psychological distress. In terms of depression status, most of the participants have mild $(\mathrm{N}=222,74 \%)$ symptoms. The level of depressive symptoms by the participants was measured using the Patient Health Questionnaire (PHQ-9). It was found that the status of the participants is mild at an average score of 7 can be described as minor symptoms. In terms of their anxiety status, most of them experienced mild anxiety $(\mathrm{N}=174,58 \%)$ and were assessed using the Generalized Anxiety Disorder Scale -7 (GAD-7), and it is found that the average respondents' have mild anxiety at an average score of 6. Table 3.2.1 shows that the physical activity engagement of the students and their psychological distress status, depression, and anxiety are negatively significant. Although some participants experienced mild depression and anxiety, the result reveals that when they perform $100 \%$ in their classroom physical activities, they do not likely experience psychological distress, depression, and anxiety. Therefore, the Affective Reflective Theory posits that, in the face of an exercise-related stimulus, if the affective valuation is positive, it will present a driving force and thus make it more likely to that person will change his or her current state of physical inactivity. Hence, physical activities that are given by the teacher act as a positive driving force that alleviates the psychological distress status of the students. This confirms that exercise effectively reduces fatigue, improves alertness and concentration, and enhances overall function. This can help when stress has depleted the energy or ability to concentrate. Exercise and physical activity produce endorphins - chemicals in the brain that acts as natural painkillers. It improved the ability to sleep, reducing stress (ADAA, 2022). In addition, exercise can help with depression, anxiety, and ADHD. One's enthusiasm towards physical fitness can start with a bit of exercise that will eventually improve one's mental health, vitality, and outlook (Robinson et al., 2020).

\subsection{The Respondents Physical Exercise Engagement and Mental Health Status}

Table 3.3.1 Correlation Between the Respondents Physical Exercise Engagement and Mental Health Status

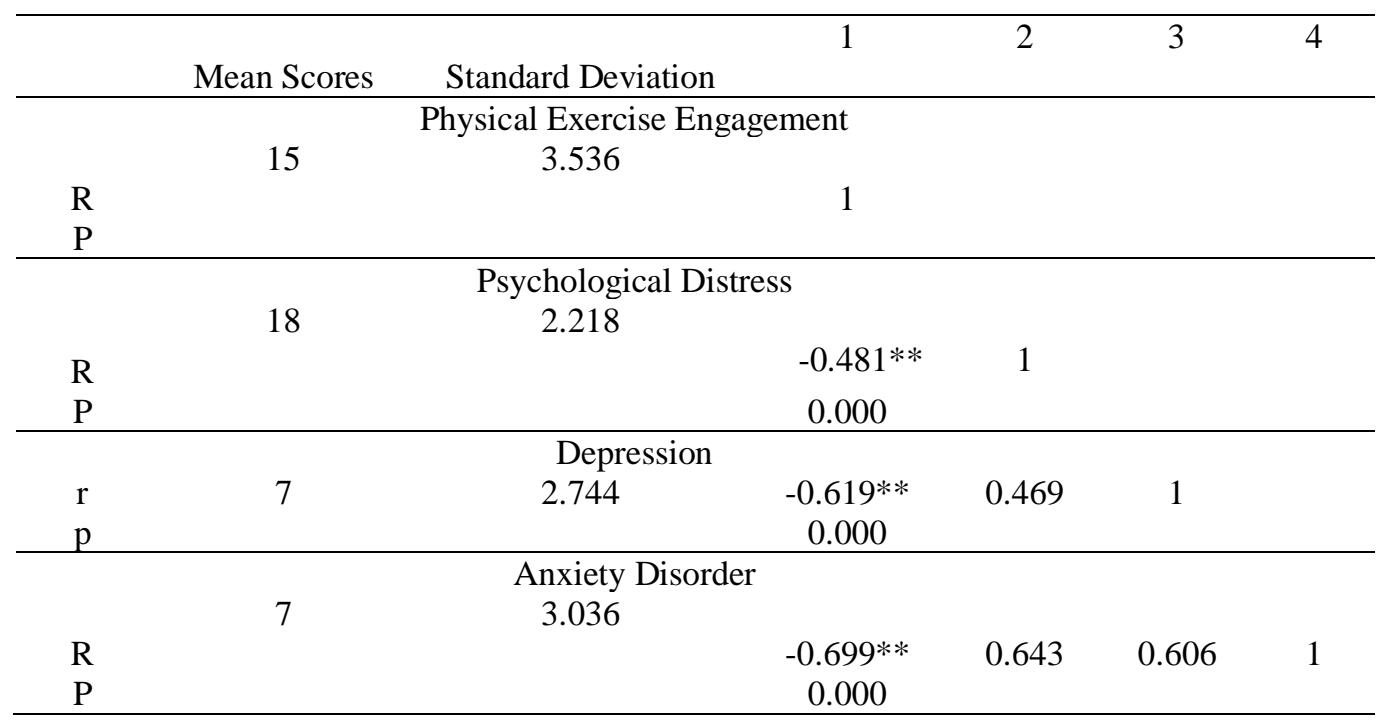




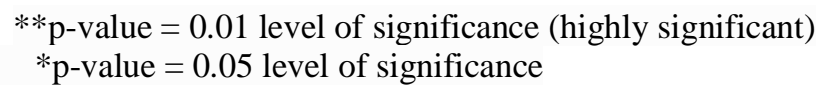

The table 3.3.1 shows the significant correlation of physical exercise engagement to mental health status to the respondents. Table 4.3.1 reveals that physical exercise has a low negative correlation to psychological distress at $r=-0.481$, and there is a significant relationship at $p=0.000$. It also shows that physical exercise has a moderate negative correlation to depression severity at $\mathrm{r}=$ 0.619 , and there is a significant relationship at $\mathrm{p}=0.000$. It further reveals that physical exercise has a moderate negative correlation to anxiety disorder at $\mathrm{r}=-0.699$, and there is a significant relationship at $\mathrm{p}=0.000$. It further implies that the more an individual engages in physical exercise, the less likely he/she experience psychological distress, depression symptoms, and anxiety symptoms. Ai et al. (2021) affirmed that the positive relationship between physical exercise and mental health has been well-established. Mainly supervised exercises are conducive to enhancing happiness and improving mental health; physical exercise reduces anxiety, sadness, and depression. Maintenance and improvement of mental health were related to the intensity and frequency of physical exercise (Ai et al., 2021). Chtourou et al. (2020) cited that staying physically active even in the pandemic would help maintain and improve mental health.

Moreover, maintaining and engaging in regular exercise is effective, therefore, an essential strategy to maintain well-being (Maugeri et al., 2020). The research found a correlation between the frequency of regular physical exercise and mental health (Chekroud et al., 2019). The connection is: that the more one regularly exercises, the better the mental health of the said individual will be. The research also found an optimal range for physical exercise frequency and duration. When youth are involved in sports and any physical activity, they will have a quality experience their mental health also improves (Elbe et al., 2019).

\section{Conclusion}

This study examines the impact of physical exercise engagement on the mental health statuses such as psychological distress, depression, and anxiety. This study determines the sociodemographic profile of the participants. Their level of physical exercise engagement and mental health status were assessed through Kessler Psychological Distress Scale, Patient Health Questionnaire, and Generalized Anxiety Disorder Scale (GAD-7). Furthermore, the correlation between the two variables were also determined. A total of 300 have responded to the online survey through google form using a modified questionnaire appropriate in measuring the variables.

The Affective Reflective Theory (Brand and Ekkekakis, 2018) supported this study and describes that the concept of the is a dual-process theory. It explains that when the affective valuation is positive, it will present a driving force and thus make it more likely that the person will change his or her current state of physical inactivity (Brand et al., 2019). Physical Educators encourage students to perform physical activity that will serve as a stimulus to activate and trigger an automatic affective valuation. Thus, the study's general findings showed that the more students engage in physical activity, the less likely they experience psychological distress, depression, and anxiety. So, they need to engage in physical activity to overcome environmental variables, such as interpersonal stress, that can contribute to chronic stress and anxiety, such as post-traumatic stress disorder (PTSD) (Mental Health.Gov, 2021). Therefore, to overcome the impact of COVID-19 on mental health, exercise intensity should be tailored for all students. Young individuals must be engaged in physical activities to cope with mental health issues brought about by this pandemic. 


\section{References}

Anxiety and Depression Association of America (2022). Physical activity reduces stress. https://adaa.org/understandinganxiety/related-illnesses/other-related-conditions/stress/physical-activity-reduces-st

Brand and Ekkekakis (2018). Theories to Explain Exercise Motivation and Physical Inactivity: Ways of Expanding Our Current Theoretical Perspective. Retrieved from Frontiers in Psychology website: https://www.frontiersin.org/articles/10.3389/ fpsyg.2019.01147/full

Brand, Ralf and Cheval, Boris. (2019). Theories to explain exercise motivation and physical inactivity: Ways of expanding our current theoretical perspective. Front. Psychol., 21 May 2019| https://doi.org/10.3389/fpsyg.2019.01147

Chtourou, H., Trabelsi, K., H'mida, C., Boukhris, O., Glenn, J. M., Brach, M., et al. (2020). Staying physically active during the quarantine and self-isolation period for controlling and mitigating the COVID-19 pandemic: a systematic overview of the literature. Front. Psychol. 11:1708. doi: 10.3389/fpsyg.2020.01708

Chekroud SR, Gueorguieva R, Zheutlin AB, Paulus M, Krumholz HM, Krystal JH. Association between physical exercise and mental health in 1.2 million individuals in the USA between 2011 and 2015: a cross-sectional study. Lancet Psychiatr. 2018; 5:739-746.

Elbe, A.M., Lyhne, S. N., Madsen, E. E., \& Krustrup, P. (2019). Is regular physical activity a key to mental health? commentary on association between physical exercise and mental health in 1.2 million individuals in the usa between 2011 and 2015 : a crosssectional study" by chekroud et al., published in lancet psychiatry. J Sport Health Sci. 2019 Jan; 8(1): 6-7. Published online 2018 Nov 22. doi: 10.1016/j.jshs.2018.11.005

Gupta, A., Puyat, J. H., Ranote, H., Vila-Rodriguez, F., and Kazanjian, A. (2021). A cross-sectional survey of activities to support mental wellness during the COVID-19 pandemic. J. Affect. Disord. Rep. 5:100167. doi: 10.1016/j.jadr.2021.100167 Henrique et al (2017). Engagement in physical education classes and health among young people: does sports practice matter? A cross-sectional study. Retrieved from Scielo Brzil website: https://www.scielo.br/j/spmj/a/wryQmwWhxt9scW3sPswHmPq /?lang=en

IGI Global (2021) What is Students. Retrieved from IGI Global, Publisher of Timely Knowledge website: https://www.igiglobal.com/dictionary/students/47331

Kessler RC, Barker PR, Colpe LJ, Epstein JF, Gfroerer JC, Hiripi E, et al. (2003), Screening for serious mental illness in the general population, Kessler Psychological Distress Scale (K10) Retrieved from

https://www.worksafe.qld.gov.au/_data/assets/pdf_file/0010/22240/kessler-psychological-distress-scale-k101.pdf

Kurt Kroenke, MD (2001), PHQ-9 (Patient Health Questionnaire-9) Retrieved from MD+ CALC website:

https://www.mdcalc.com/phq-9-patient-health-questionnaire-9

Kurt Kroenke, MD, Oviahealth (2021), Interpreting your PHQ-9 depression screening results. Retrieved from ovia health website: https://www.oviahealth.com/guide/107365/fertility-pep-understanding-phq9/

Lawalata, Imanuel (2021). The benefits of regular exercise. https://www.imanuellawalata.com/the-benefits-of-regular-exercise/

Maugeri, G., Castrogiovanni, P., Battaglia, G., Pippi, R., D'Agata, V., Palma, A., et al. (2020). The impact of physical activity on psychological health during Covid-19 pandemic in Italy. Heliyon 6:e04315. doi: 10.1016/j.heliyon.2020.e04315

MentalHealth.gov, (2021). What is Mental health? Retrieved from MentalHealth.gov Let's Talk about it website: https://www.mentalhealth.gov/basics/what-is-mental-health

Richard Weil, MEd, CDE and Melissa Conrad Stöppler, MD (2021). Aerobic exercise: types, list and benefits. Retrieved from MedicineNet website: https://www.medicinenet.com/aerobic_exercise/article.htm

Robinson, L., Segal, J., and Smith, M. (2020). Exercise and Fitness, The mental health benefits of exercise. Retrieved from Help Guide website: https://www.helpguide.org/articles/healthy-living/the-mental-health-benefits-of-exercise.htm

Robinson, Lawrence et Al (2021). The Mental Health Benefits of Exercise. Retrieved from Exercise and Fitness website: https://www.helpguide.org/articles/healthy-living/the-mental-health-benefits-of-exercise.htm

Sascha Leisterer and Darko Jekauc. Students' Emotional Experience in Physical Education-A Qualitative Study for New Theoretical Insights. Sports. 2019; 7(1):10. https://doi.org/10.3390/sports7010010

Unicef Philippines (2021). COVID-19: Schools for more than 168 million children globally have been completely closed for almost a full year, says UNICEF

Theguardian.com (2021). Covid crisis dramatically worsened global mental health.

https://www.theguardian.com/world/2021/oct/08/covid-crisis-dramatically-worsened-global-mental-health-study-finds

Warburton, D. E., Nicol, C. W., and Bredin, S. S. (2006). Health benefits of physical activity: the evidence. CMAJ 174, 801-809. doi: $10.1503 / \mathrm{cmaj} .051351$

Weil, et. al. (2021). The Subjective Meaning of Sleep Quality: A Comparison of Individuals with and without Insomnia. Retrieved from US National Library of Medicine National Institutes of Health website: https://www.ncbi.nlm.nih.gov/pmc/articles/PMC2276747/

World Health Organization (2019). WHO-led Study on Insufficient Physical Activity among Adolescents leaves out LGBTQI2S Youth. https://egale.ca/wp-content/uploads/2019/12/Egale_Response-to-WHO-led-Study_LGBTQI2SSport.pdf

Xianfeng Ai, Jingjing Yang, Zhibin Lin, and Xiaohong Wan (2021). Mental health and the role of physical activity during the COVID-19 Pandemic. Front. Psychol., https://doi.org/10.3389/fpsyg.2021.759987 